\title{
Why an intrinsic magnetic field does not protect a planet against atmospheric escape
}

\author{
Herbert Gunell ${ }^{1,2}$, Romain Maggiolo ${ }^{1}$, Hans Nilsson ${ }^{3}$, Gabriella Stenberg Wieser ${ }^{3}$, Rikard Slapak ${ }^{4}$, \\ Jesper Lindkvist ${ }^{2}$, Maria Hamrin ${ }^{2}$, and Johan De Keyser ${ }^{1}$ \\ ${ }^{1}$ Royal Belgian Institute for Space Aeronomy (BIRA-IASB), Avenue Circulaire 3, 1180 Brussels, Belgium \\ e-mail: herbert . gunell@physics .org \\ 2 Department of Physics, Umeå University, 90187 Umeå, Sweden \\ 3 Swedish Institute of Space Physics, Box 812, 98128 Kiruna, Sweden \\ ${ }^{4}$ EISCAT Scientific Association, P. O. Box 812, 98128 Kiruna, Sweden
}

Received 2 March 2018 / Accepted 23 May 2018

\begin{abstract}
The presence or absence of a magnetic field determines the nature of how a planet interacts with the solar wind and what paths are available for atmospheric escape. Magnetospheres form both around magnetised planets, such as Earth, and unmagnetised planets, like Mars and Venus, but it has been suggested that magnetised planets are better protected against atmospheric loss. However, the observed mass escape rates from these three planets are similar (in the approximate $(0.5-2) \mathrm{kg} \mathrm{s}^{-1}$ range), putting this latter hypothesis into question. Modelling the effects of a planetary magnetic field on the major atmospheric escape processes, we show that the escape rate can be higher for magnetised planets over a wide range of magnetisations due to escape of ions through the polar caps and cusps. Therefore, contrary to what has previously been believed, magnetisation is not a sufficient condition for protecting a planet from atmospheric loss. Estimates of the atmospheric escape rates from exoplanets must therefore address all escape processes and their dependence on the planet's magnetisation.
\end{abstract}

Key words. Planets and satellites: magnetic fields - Planets and satellites: atmospheres - plasmas

\section{Introduction}

Due to the interaction between the planetary environment and the solar wind, a boundary region is formed around both magnetised and unmagnetised planets. This creates an obstacle to the solar wind flow, deflecting most of it. At a magnetised planet, the magnetosphere is the part of space dominated by the planetary magnetic field, and the magnetopause is the outer boundary of the magnetosphere (Fig. 1a). Around an unmagnetised planet with an atmosphere (Fig. 1b), an induced magnetosphere is formed (Russell 1993). Its outer boundary is called the induced magnetosphere boundary (IMB; Lundin et al. 2004). The ionospheres of the unmagnetised planets are not magnetically connected to the solar wind, but the IMB is located closer to the planet than the magnetopause is to a magnetised planet. Observations of their magnetic topologies put Venus and Mars in the unmagnetised and Earth in the magnetised category (Russell 1993) (see Appendix A.1 for a mathematical description of the distinction between magnetised and unmagnetised planets).

These differences in magnetic topology affect the atmospheric escape processes at work (Lundin et al. 2007). What we can learn about the role of planetary magnetisation in protecting the atmospheres of planets in our solar system will form the foundation for our understanding of how atmospheres of magnetised and unmagnetised planets in other solar systems evolve. This has major implications for the assessment of the habitability of these exoplanets. We estimate the escape rates of the major atmospheric escape processes and their dependencies on various factors using a parameterised model, describing the escape rates as functions of planetary magnetic dipole moment. The results are applied to the terrestrial planets, computing escape rates for hypothetical Venus-like, Earth-like, and Marslike planets that have the atmospheric properties these planets have today. Venus, Earth, and Mars are all rocky planets with atmospheres for which satellite-based measurements of atmospheric escape are available (e.g., Barabash et al. 2007; Strangeway et al. 2005; Lundin et al. 2004). For present-day conditions, the escape rates we arrive at in this work are about $0.5 \mathrm{~kg} \mathrm{~s}^{-1}$ for Venus, $1.4 \mathrm{~kg} \mathrm{~s}^{-1}$ for Earth, and between $0.7 \mathrm{~kg} \mathrm{~s}^{-1}$ and $2.1 \mathrm{~kg} \mathrm{~s}^{-1}$ for Mars (Fig. 2a). We specify a range rather than a single number for Mars because of the uncertainties in the escape rate caused by dissociative recombination of molecular oxygen ions (Appendix A.2). Earth's magnetic moment is $7.77 \times 10^{22} \mathrm{~A} \mathrm{~m}^{2}$, while measurements at the unmagnetised planets Mars and Venus have shown that their magnetic moments are below $2 \times 10^{18} \mathrm{~A} \mathrm{~m}^{2}$ and $7 \times 10^{18} \mathrm{~A} \mathrm{~m}^{2}$, respectively (Acuña et al. 2001; Olson \& Amit 2006; Luhmann et al. 2015) (V, $\mathrm{M}$ and $\mathrm{E}$ in Fig. 2). For reference, the magnetic moment of Jupiter, a highly magnetised planet with a magnetic moment of $1.56 \times 10^{27} \mathrm{~A} \mathrm{~m}^{2}$ (Guillot et al. 2004), is indicated in the diagram. However, Jupiter's magnetosphere is a more complicated system, with additional internal plasma sources driven by its moons, and the model used here is not applicable to that planet.

The escape rates depend on the solar ultraviolet (UV) flux and the properties of the atmosphere and solar wind, and these conditions may change over the lifetime of a planetary system 
a Magnetised planet

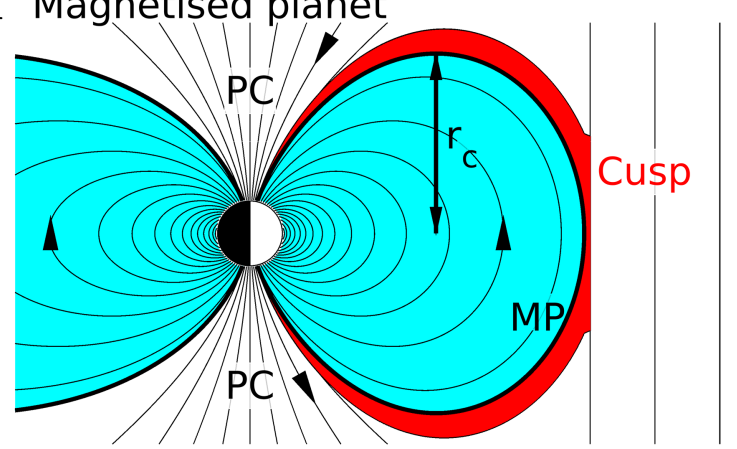

b Unmagnetised planet

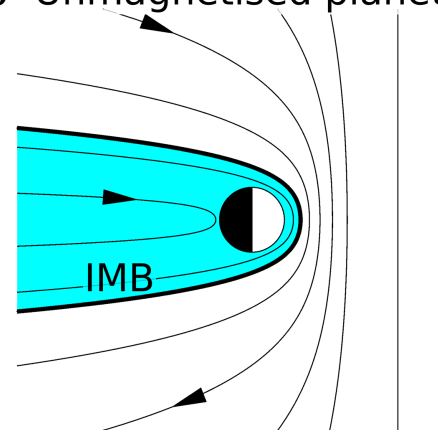

Fig. 1. Magnetic configuration. Schematic drawings of the magnetic configurations of magnetised and unmagnetised planets. Panel a: magnetised planet with the magnetopause (MP), the polar caps (PC), and the cusps shown in red. The maximum distance between the dayside MP and the planet-sun line, $r_{\mathrm{c}}$, is used in the model (Appendix A). Panel $b$ : unmagnetised planet showing the induced magnetosphere boundary (IMB).

(Lammer 2013). Other planetary properties may be correlated with the presence of a magnetic field; for example, planetary size and rotation may affect both the magnetic dynamo and, via plate tectonics, the density and composition of the atmosphere. We emphasise the physical effects of planetary magnetic fields, considering only the average present-day solar conditions and using the average present-day values of planetary parameters other than the magnetic moment. We consider only hydrogen and oxygen, since these are the most populous exospheric species.

\section{Escape processes}

A number of different processes contribute to atmospheric escape: Jeans escape, dissociative recombination, cross-field ion loss, ion pickup, sputtering, and escape through the cusp and the polar cap. We model each of these, bringing them together to obtain the total escape rate as a function of planetary magnetic moment (see Appendix A.2 for calculation details). Two of the modelled processes are independent of the magnetic moment, and their contributions are included as constants (Appendix A.2): Jeans escape (Öpik 1963), where the tail of the thermal distribution reaches escape energy, and dissociative recombination of oxygen molecules (Cravens et al. 2017), which produces oxygen atoms with energies above the escape energy of Mars but not above that of the heavier planets.

Cross-field ion loss, in which ions are transported slowly across magnetic field lines, eventually escaping, depends only weakly on the magnetic moment. Since ions are seen to migrate across field lines on all the terrestrial planets - through the plasmaspheric wind and plumes at Earth (Lemaire \& Schunk 1992; André \& Cully 2012) and directly from the ionosphere at Mars and Venus (Lundin et al. 2008; Nordström et al. 2013;

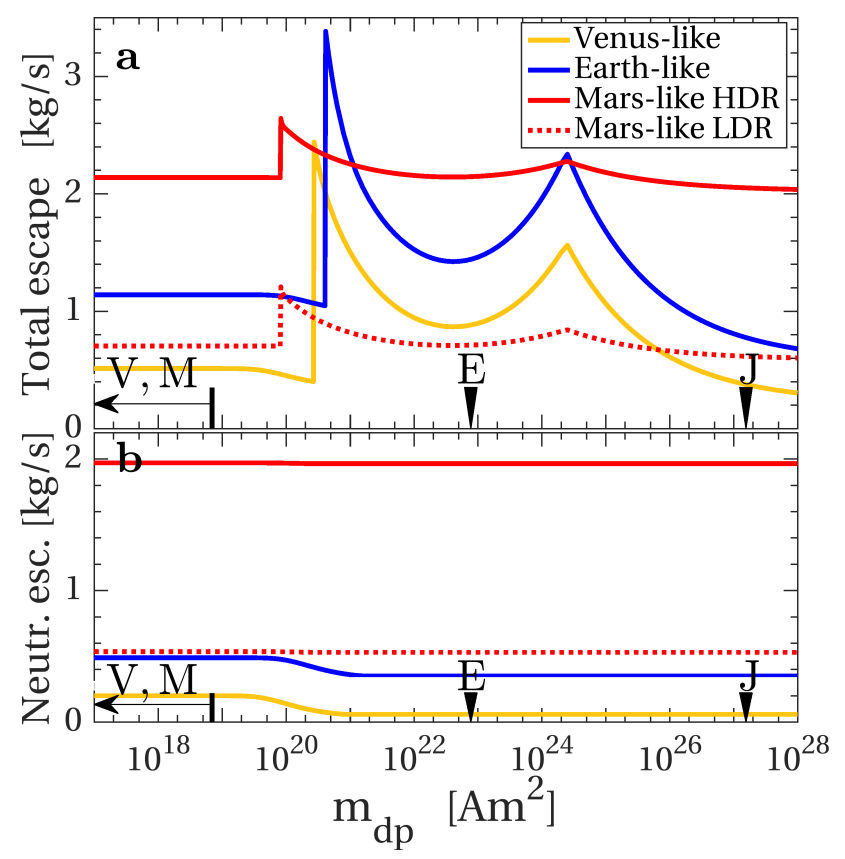

Fig. 2. Mass escape rates for Venus-like, Earth-like, and Mars-like planets. Panel $a$ : total mass escape rates. Panel $b$ : neutral mass escape rates. For escape from Mars, the two curves represent high (HDR) and low (LDR) estimates of dissociative recombination of molecular oxygen. The magnetic moments of present-day Earth and Jupiter are marked on the horizontal axis for reference. The horizontal arrow indicates the unmagnetised character of Venus and Mars.

Edberg et al. 2011) - and, on average, escape is unhindered by the magnetic field, we treat cross-field ion loss as one single process, although the microphysical processes behind it are likely to be different at the different planets.

In ion pickup, atoms of the exosphere located outside the MP or IMB are ionised and picked up by the solar wind. Its contribution is significant in the $10^{24}-10^{25} \mathrm{~s}^{-1}$ range for Mars and Venus (Lammer 2013; Ramstad et al. 2015; Masunaga et al. 2013), but decreases rapidly when the magnetosphere grows with increasing magnetic moment (Appendix A.2 and Fig. 3). It is therefore an insignificant process for Earth. For an unmagnetised Earthlike planet, it would be significant for hydrogen but not oxygen. The difference between the species, and between the planets, is largely determined by the scale height, which depends on the temperature and atomic mass of the species as well as the mass of the planet (see Table A.1 for numerical values).

Atmospheric sputtering is caused by ionospheric ions swept up and accelerated by the solar wind that then reimpact the ionosphere, colliding with exospheric oxygen atoms, giving these enough energy to escape the planet (Luhmann \& Kozyra 1991). The ion gyroradius decreases with an increasing magnetic field. Therefore, the region from which newborn ions can reach the exobase shrinks with increasing planetary magnetic moment. Also, the magnetosphere shields this region from the convective electric field of the solar wind. Sputtering therefore decreases with an increasing magnetic moment (Fig. 3). The gyroradius is larger for oxygen ions than for protons, and the sputtering efficiency is higher for ions sputtering their own neutrals. Therefore, only oxygen ions sputtering neutral oxygen are significant. The sputtering escape rate is $6 \times 10^{24} \mathrm{~s}^{-1}$ for Venus (Luhmann \& Kozyra 1991; Nordström et al. 2013), and would be similar for unmagnetised Earth-like planets, while it is at most $5 \times 10^{23} \mathrm{~s}^{-1}$ for Mars (Chaufray et al. 2007). Yet, Jakosky et al. (2017) speculated that sputtering could have been a significant 


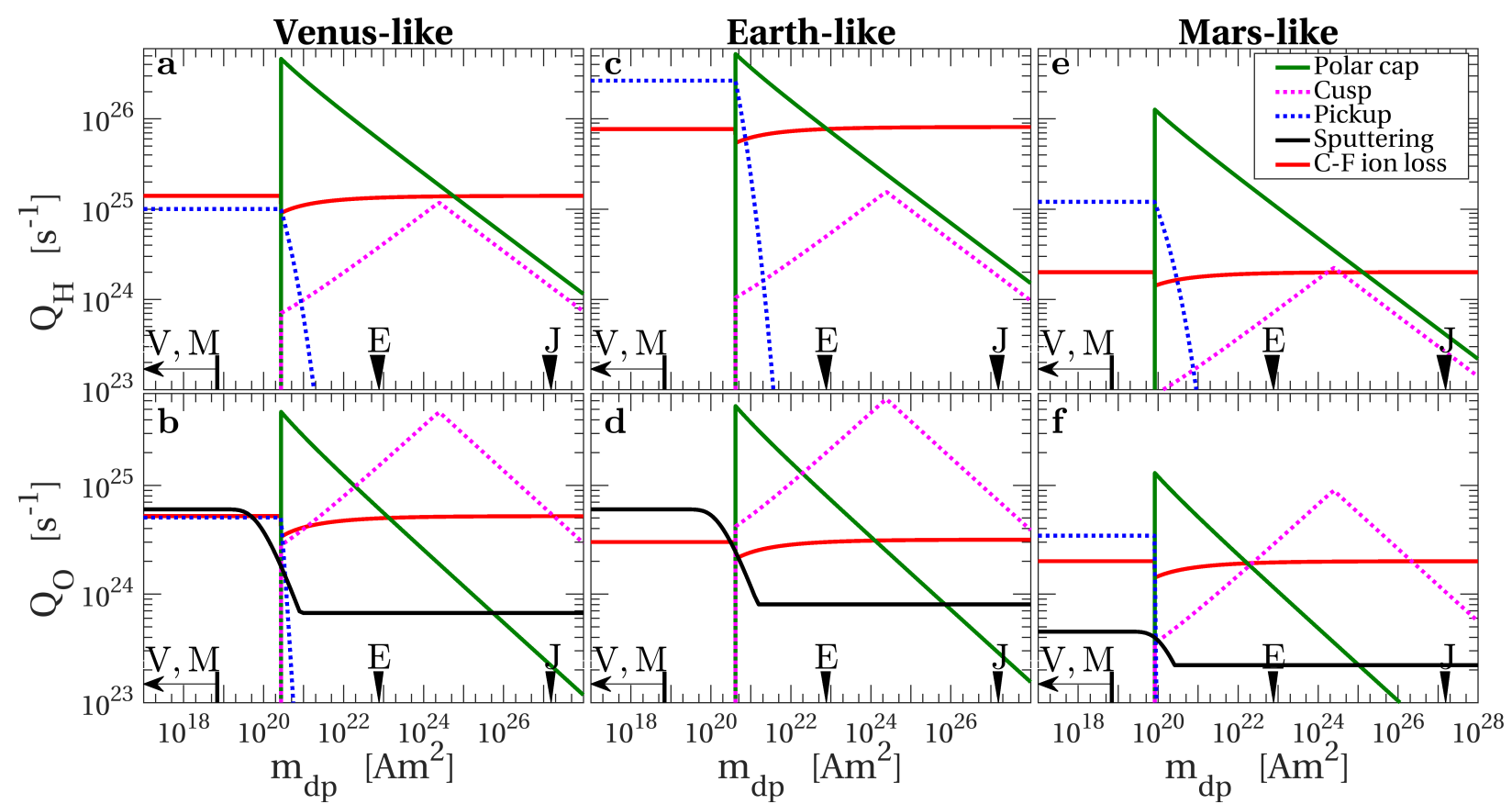

Fig. 3. Escape rates for different processes. Escape rates are shown for the processes that depend on the planetary magnetic moment. Hydrogen escape rates are shown in panels $a$, $c$, and $e$, and oxygen escape rates in panels $b, d$, and $f$. Venus-like planets are shown in panels $a$ and $b$; Earth-like in panels $c$ and $d$ and Mars-like in panels $e$ and $f$. The magnetic moments of present-day Earth and Jupiter are marked on the horizontal axis for reference. The horizontal arrow indicates the unmagnetised character of Venus and Mars.

escape mechanism for Mars in the past. The sputtering rate falls off quickly with increasing magnetic moment as a planet enters the magnetised range (Fig. 3). Escape rates from sputtering caused by precipitation in the auroral zone have been estimated to be $8 \times 10^{23} \mathrm{~s}^{-1}$ (Shematovich et al. 2006). This can be seen as a lower limit of the sputtering escape rate, but it is negligible in comparison with other processes.

The polar caps are the regions around the magnetic poles where the magnetic field lines are open, that is to say, where they have one end in the ionosphere and the other in interplanetary space (Fig. 1). In the polar cap, plasma escapes by means of a polar wind (Axford 1968). The electrons are faster than the ions, and therefore set up an ambipolar electric field, accelerating ions upward. The upward flux (ions $\mathrm{m}^{-2} \mathrm{~s}^{-1}$ ) depends on solar illumination, which affects the properties of the ionosphere. The flux is small and steady compared to that in the cusps (Maes et al. 2016), but the area of the polar cap is larger than that of the cusp by an order of magnitude. The escape rate scales with the area of the polar caps (defined in Appendix A.1). It is highest for the magnetic moment just above the transition from an unmagnetised to a magnetised planet, where the polar cap is at its maximum size, and for hydrogen this is the highest peak (Fig. A.1). The polar cap escape rate then falls off with increasing magnetic moment. For an unmagnetised planet, there is no polar cap. To define the transition between the magnetised and unmagnetised states we compare the MP standoff distance to the standoff distance of the IMB (see Appendix A.1 for details).

The cusp is the part of the polar cap that maps magnetically to the dayside MP (Fig. 1). In that region, solar wind plasma first flows planetward along the magnetic field and then reverses direction due to the magnetic mirror force. Kinetic energy is carried into the cusp by this plasma, and energy can also be transferred by a Poynting flux in the same direction (Strangeway et al. 2005). This energy contributes to heating of ionospheric particles leading to their subsequent escape. For low magnetic moments, the escape rate from the cusp is proportional to the area of the cusp and to the incident energy flux (Moore \& Khazanov 2010), which increases with the cross section of the magnetosphere $\pi r_{\mathrm{c}}^{2}$, where $r_{\mathrm{c}}$ is the maximum distance to the planet-sun line of the dayside MP (Appendix A.1). Therefore, the escape rate increases with magnetic moment until a point where the maximum ion flux that the ionosphere can supply is reached (Barakat et al. 1987) (Appendix A.2). For higher magnetic moments, the upward flux becomes saturated when it reaches the maximum that can be delivered by the ionosphere. The escape rate then decreases as the area of the cusp decreases. This causes a peak near a magnetic moment of $2.5 \times 10^{24} \mathrm{~A} \mathrm{~m}^{2}$ (Fig. 3), about thirty times the magnetic moment of present-day Earth.

\section{Conclusions and discussion}

We show that the mass escape rate, including both oxygen and hydrogen (Fig. 2a), for each kind of planet is similar in the unmagnetised range and for high magnetisations, that is, with magnetic moments of the same magnitude as that of Jupiter. In between, there are two maxima. The first corresponds to polar cap escape and is dominant for hydrogen, while the second peak is dominated by cusp escape. The escape peaks are higher than the unmagnetised escape by a factor in the range 3-5 for Venus-like planets, 2-3 for Earth-like and less than 2 for Mars-like planets. The peaks are caused by ion escape processes, while the combined rates for neutral escape processes, that is, Jeans escape, dissociative recombination, and sputtering, shown in Fig. 2b, are mostly flat.

The mass escape rate from present-day, magnetised, Earth is somewhat higher than from an Earth-like unmagnetised planet. The same can be said for Mars-like and Venus-like planets. With the current outflow rate of $1 \mathrm{~kg} \mathrm{~s}^{-1}$, less than $1 \%$ of Earth's atmosphere is removed in a gigayear (André 2015). Even the maximum escape rates in Fig. $2 \mathrm{a}$ of $\sim 3 \mathrm{~kg} \mathrm{~s}^{-1}$, cannot have removed as much as one Earth atmosphere from any of the terrestrial planets in the time since their formation. However, higher 
solar wind pressure and EUV flux in the early solar system could have led to an expansion of the exospheres of the planets and, subsequently, higher escape rates (Lammer 2013), but such an exospheric expansion would lead to higher escape rates from both magnetised and unmagnetised planets. For example, an expanded exosphere would lead to an increased cusp area, and higher EUV flux would increase ion production and thus raise the limiting fluxes that determine the height of the cusp escape peak (Figs. 2a and 3). Previously, models of atmospheric escape from exoplanets that only account for ion pickup and Jeans escape (Lammer et al. 2007) have indicated that magnetisation is necessary for habitability; it is possible that this picture will change when cusp and polar-cap escape processes, active at magnetised planets, are included.

It has been argued that a significant number of the oxygen ions leaving Earth through the cusps and polar caps return to the atmosphere (Seki et al. 2001). However, it was later shown that the energy of the upflowing heavy ions is high enough for them to escape directly to interplanetary space, and that the return flows are negligible (Nilsson 2011; Nilsson et al. 2012). This has been confirmed in recent studies (Slapak et al. 2017a,b). For hydrogen, the return flows are sufficient to make the magnetospheric $\mathrm{H}^{+}$budget more stable, but there is still a net loss of $7.8 \times 10^{25} \mathrm{~s}^{-1}$ (Slapak et al. 2017a). The hydrogen peak at the transition between magnetised and unmagnetised planets could be smoothed out by processes unknown to us, as that regime is observationally inaccessible. However, due to the 16 times larger mass of the $\mathrm{O}^{+}$ions, this would not change our conclusions about the total mass escape.

While a planetary magnetic field protects the atmosphere from sputtering and ion pickup, it enables polar cap and cusp escape, which increases the escape rate. Furthermore, the induced magnetospheres of the unmagnetised planets also provide protection from sputtering and ion pickup in the same way as the magnetospheres of the magnetised planets. Therefore, contrary to what has been believed and reported in the press (Achenbach 2017), the presence of a strong planetary magnetic field does not necessarily protect a planet from losing its atmosphere. While we model the overall behaviour, the current understanding of the details of several escape mechanisms can still be improved, by future theoretical and observational studies. When assessing the habitability of planets in the emerging field of exoplanet research, it is essential to properly assess the role an intrinsic magnetic field plays in atmospheric protection.

Acknowledgements. Work at the Royal Belgian Institute for Space Aeronomy was supported by the Belgian Science Policy Office through the Solar-Terrestrial Centre of Excellence, and by PRODEX/Cluster contract 13127/98/NL/VJ(IC)PEA90316. Work at Umeå University was funded by SNSB grant 201/15. Work at EISCAT Scientific Association was funded by SNSB grant 141/13. Work at the Swedish Institute of Space Physics was supported by SNSB grant 96/15.

\section{References}

Achenbach, J. 2017, How Mars lost its atmosphere, and why Earth didn't, The Washington Post, 30 March

Acuña, M. H., Connerney, J. E. P., Wasilewski, P., et al. 2001, J. Geophys. Res., 106,23403

Anderson Jr., D. E., \& Hord, C. W. 1977, Planet. Space Sci., 25, 563

André, M. 2015, Phys. Scr., 90, 128005

André, M., \& Cully, C. M. 2012, Geophys. Res. Lett., 39, 3101

Axford, W. I. 1968, J. Geophys. Res., 73, 6855

Barabash, S., Fedorov, A., Sauvaud, J.-A., et al. 2007, Nature, 450, 650

Barakat, A. R., Schunk, R. W., Moore, T. E., \& Waite Jr., J. H. 1987, J. Geophys Res., 92, 12255

Bertucci, C., Duru, F., Edberg, N., et al. 2011, Space Sci. Rev., 162, 113

Chamberlain, J. W., \& Hunten, D. M. 1987, Theory of Planetary Atmospheres An Introduction to Their Physics and Chemistry, 2nd edn., International Geophysics Series (Orlando: Academic Press, Inc.)
Chaufray, J. Y., Modolo, R., Leblanc, F., et al. 2007, J. Geophys. Res. (Planets), 112, E09009

Chaufray, J. Y., Leblanc, F., Quémerais, E., \& Bertaux, J. L. 2009, J. Geophys. Res. (Planets), 114, E02006

Cravens, T. E., Rahmati, A., Fox, J. L., et al. 2017, J. Geophys. Res. (Space Phys.), 122, 1102

Edberg, N. J. T., Nilsson, H., Futaana, Y., et al. 2011, J. Geophys. Res. (Space Phys.), 116, A09308

Engwall, E., Eriksson, A. I., Cully, C. M., et al. 2009, Nat. Geosci., 2, 24

Fränz, M., Dubinin, E., Andrews, D., et al. 2015, Planet. Space Sci., 119, 92

Grießmeier, J.-M., Stadelmann, A., Penz, T., et al. 2004, A\&A, 425, 753

Guillot, T., Stevenson, D. J., Hubbard, W. B., \& Saumon, D. 2004, in Jupiter. The Planet, Satellites and Magnetosphere, eds. F. Bagenal, T. E. Dowling, \& W. B. McKinnon (Cambridge, UK: Cambridge University Press), 35

Jakosky, B. M., Slipski, M., Benna, M., et al. 2017, Science, 355, 1408

Lammer, H. 2013, Origin and Evolution of Planetary Atmospheres: Implications for Habitability, Springer Briefs in Astronomy (Berlin: Springer)

Lammer, H., \& Bauer, S. J. 1991, J. Geophys. Res., 96, 1819

Lammer, H., Lichtenegger, H. I. M., Biernat, H. K., et al. 2006, Planet. Space Sci., 54, 1445

Lammer, H., Lichtenegger, H. I. M., Kulikov, Y. N., et al. 2007, Astrobiology, 7, 185

Lemaire, J., \& Schunk, R. W. 1992, J. Atmos. Terr. Phys., 54, 467

Lichtenegger, H. I. M., Lammer, H., Kulikov, Y. N., et al. 2006, Space Sci. Rev., 126,469

Luhmann, J. G., \& Kozyra, J. U. 1991, J. Geophys. Res., 96, 5457

Luhmann, J. G., Ma, Y. J., Villarreal, M. N., Wei, H. Y., \& Zhang, T. L. 2015, Planet. Space Sci., 119, 36

Lundin, R., Barabash, S., Andersson, H., et al. 2004, Science, 305, 1933

Lundin, R., Lammer, H., \& Ribas, I. 2007, Space Sci. Rev., 129, 245

Lundin, R., Barabash, S., Holmström, M., et al. 2008, Geophys. Res. Lett., 35 , L18203

Lundin, R., Barabash, S., Holmström, M., et al. 2009, Geophys. Res. Lett., 36, L17202

Maes, L., Maggiolo, R., \& De Keyser, J. 2016, Ann. Geophys., 34, 961

Masunaga, K., Futaana, Y., Stenberg, G., et al. 2013, Geophys. Res. Lett., 40, 1682

McElroy, M. B., Prather, M. J., \& Rodriguez, J. M. 1982, Geophys. Res. Lett., 9, 649

Moore, T. E., \& Khazanov, G. V. 2010, J. Geophys. Res. (Space Phys.), 115, A00J13

Nagy, A. F., Cravens, T. E., Yee, J.-H., \& Stewart, A. I. F. 1981, Geophys. Res. Lett., 8, 629

Newell, P. T., Sotirelis, T., \& Wing, S. 2009, J. Geophys. Res. (Space Phys.), 114, A09207

Nilsson, H. 2011, in The Dynamic Magnetosphere, eds. W. Liu, \& M. Fujimoto (Dordrecht: Springer Netherlands), 315-327

Nilsson, H., Barghouthi, I. A., Slapak, R., Eriksson, A. I., \& André, M. 2012, J. Geophys. Res. (Space Phys.), 117, A11201

Nilsson, H., Carlsson, E., Gunell, H., et al. 2006, Space Sci. Rev., 126, 355

Nilsson, H., Edberg, N. J. T., Stenberg, G., et al. 2011, Icarus, 215, 475

Nordström, T., Stenberg, G., Nilsson, H., Barabash, S., \& Zhang, T. L. 2013, J. Geophys. Res. (Space Phys.), 118, 3592

Olson, P., \& Amit, H. 2006, Naturwissenschaften, 93, 519

Öpik, E. J. 1963, Geophys. J. R. Astron. Soc., 7, 490

Pollock, C. J., Chandler, M. O., Moore, T. E., et al. 1990, J. Geophys. Res., 95, 18969

Ramstad, R., Barabash, S., Futaana, Y., et al. 2015, J. Geophys. Res. (Planets), 120, 1298

Russell, C. T. 1993, Rep. Prog. Phys., 56, 687

Seki, K., Elphic, R. C., Hirahara, M., Terasawa, T., \& Mukai, T. 2001, Science, 291, 1939

Shematovich, V. I., Bisikalo, D. V., \& Géerard, J. C. 1994, J. Geophys. Res., 99, 23217

Shematovich, V. I., Bisikalo, D. V., \& GéRard, J.-C. 2006, J. Geophys. Res. (Space Phys.), 111, A10301

Slapak, R., Nilsson, H., \& Westerberg, L. G. 2013, Ann. Geophys., 31, 1005

Slapak, R., Hamrin, M., Pitkänen, T., et al. 2017a, Ann. Geophys., 35, 869

Slapak, R., Schillings, A., Nilsson, H., et al. 2017b, Ann. Geophys., 35, 721

Spreiter, J. R., \& Stahara, S. S. 1992, Washington DC American Geophysical Union Geophysical Monograph Series, 66, 345

Stenberg Wieser, G., Ashfaque, M., Nilsson, H., et al. 2015, Planet. Space Sci., 113,369

Strangeway, R. J., Ergun, R. E., Su, Y.-J., Carlson, C. W., \& Elphic, R. C. 2005, J. Geophys. Res. (Space Phys.), 110, A03221

Voigt, G.-H. 1981, Planet. Space Sci., 29, 1

Voigt, G.-H. 1995, in Handbook of Atmospheric Electrodynamics, 2, 333

Welling, D. T., André, M., Dandouras, I., et al. 2015, Space Sci. Rev., 192, 145 


\section{Appendix A: Model description}

We model the total escape rate by assuming seven escape processes described by parametric functions. Five of these processes are dependent on simple properties of the planetary magnetosphere, which is simulated assuming a magnetic field model and solar wind dynamic pressure balance. Each escape-rate function is defined so that it is consistent with observationally estimated rates for the specific processes listed in Table A.1.

\section{A.1. Magnetic model}

The escape processes that affect ions depend on the MP standoff distance $R_{\mathrm{MP}}$, the solid angle of the polar caps $\Omega_{\mathrm{pc}}$, and the cross section of the magnetosphere $\pi r_{\mathrm{c}}^{2}$, where $r_{\mathrm{c}}$ is the maximum distance from the dayside MP to the planet-sun line. For an unmagnetised planet, $r_{\mathrm{c}}=r_{\mathrm{IMB}}$ is the standoff distance of the induced magnetosphere boundary. For a magnetised planet, $r_{\mathrm{c}}$ is found by tracing a magnetic field line from the subsolar MP towards the planet. Assuming pressure balance between the solar wind dynamic pressure and the magnetic pressure of the magnetosphere, the standoff distance of the MP can be estimated by Voigt (1981), Grießmeier et al. (2004)

$R_{\mathrm{MP}}=\left(\frac{\mu_{0} f_{0}^{2} m_{\mathrm{dp}}^{2}}{8 \pi^{2} n_{\mathrm{sw}} v_{\mathrm{sw}}^{2}}\right)^{1 / 6}$,

where $m_{\mathrm{dp}}$ is the magnetic dipole moment of the planet, $n_{\mathrm{sw}}$ is the solar wind density, $v_{\mathrm{sw}}$ the solar wind speed, and $f_{0}=1.16$, which is a form factor that gives the MP a realistic shape (Voigt 1995). A planet is considered unmagnetised if $R_{\mathrm{MP}}<r_{\mathrm{IMB}}$. For Mars and Venus, $r_{\mathrm{IMB}}$ is given by observations (Stenberg Wieser et al. 2015; Bertucci et al. 2011); for Earth, it is estimated assuming a pressure balance between the exosphere and the solar wind (Spreiter \& Stahara 1992) and using a Chamberlain exosphere (Chamberlain \& Hunten 1987) for these low altitudes.

The magnetic field is traced back from the subsolar magnetosphere towards the planet in order to find $r_{\mathrm{c}}$ and $\Omega_{\mathrm{pc}}$. For $r_{\mathrm{c}}$, we use the Voigt magnetic model (Voigt 1981) of the dayside magnetosphere with a superimposed southward interplanetary magnetic field component included:

$\boldsymbol{B}(x, z)=\boldsymbol{B}_{\mathrm{dp}}(x, z)+\left(2 f_{0}-1\right) \boldsymbol{B}_{\mathrm{dp}}\left(R_{\mathrm{MP}}, 0\right)+\boldsymbol{B}_{\mathrm{sw}}$,

where $\boldsymbol{B}_{\mathrm{dp}}(x, z)$ is the planetary dipole field and $\boldsymbol{B}_{\mathrm{sw}}$ is the IMF $B_{z}$ component given in Table A.1. We model average outflow rates based on measurements. Therefore, we use IMF $B_{z}$ values that are typical during southward IMF, which is when most of the escape occurs. When the magnetosphere is compressed on the dayside, it is also elongated on the nightside. The polar cap is pushed toward the nightside and not uniformly contracted. Therefore, Eq. (A.2) and the assumption of a circular pole-centred polar cap would lead to an underestimate of $\Omega_{\mathrm{pc}}$. Instead we use a dipole field for finding $\Omega_{\mathrm{pc}}$. A field line is traced, in that field, from the subsolar MP down to the exobase, and the polar cap area is approximated by a circle centred on the pole at the exobase altitude. For a magnetic dipole, a field line that crosses the magnetic equator at $r=R_{\mathrm{MP}}$ intersects a sphere with radius $r=r_{\text {exo }}$ at magnetic latitude $\lambda$ given by

$1=L \cos ^{2}(\lambda)$

where $L=R_{\mathrm{MP}} / r_{\text {exo }}$ is McIlwain's $L$-parameter, here defined with the exobase radius instead of the usual planetary radius in the denominator. Therefore, $\lambda$ is found by $\lambda=\arccos \sqrt{\frac{1}{L}}$.

To find the solid angle of the polar caps, we integrate from the pole out to $\lambda$ and multiply by two, because there are two polar caps. For the magnetised range, we have

$$
\begin{aligned}
\Omega_{\mathrm{pc}}\left(r_{\mathrm{IMB}} \leq R_{\mathrm{MP}}\right) & =2 \int_{0}^{\frac{\pi}{2}-\lambda} 2 \pi \sin \theta \mathrm{d} \theta \\
& =4 \pi\left(1-\cos \left(\frac{\pi}{2}-\lambda\right)\right) \\
& =4 \pi\left(1-\cos \left(\frac{\pi}{2}-\arccos \sqrt{\frac{1}{L}}\right)\right) \\
& =4 \pi\left(1-\sin \left(\arccos \sqrt{\frac{1}{L}}\right)\right) \\
& =4 \pi\left(1-\sqrt{1-\frac{1}{L}}\right)=4 \pi\left(1-\sqrt{1-\frac{r_{\mathrm{exo}}}{R_{\mathrm{MP}}}}\right) .
\end{aligned}
$$

In the unmagnetised range, the polar cap disappears, and for all magnetic moments, we can write

$\Omega_{\mathrm{pc}}=\left\{\begin{array}{ll}4 \pi\left(1-\sqrt{1-\frac{r_{\text {exo }}}{R_{\mathrm{MP}}}}\right) & \text { for } r_{\mathrm{IMB}} \leq R_{\mathrm{MP}} \\ 0 & \text { for } r_{\mathrm{IMB}}>R_{\mathrm{MP}}\end{array}\right.$,

where $r_{\text {exo }}$ is the exobase radius. The values used for $r_{\text {IMB }}$ are found in Table A.1.

\section{A.2. Processes}

\section{A.2.1. Jeans escape}

In Jeans escape (Öpik 1963), the atoms in the tail of the thermal distribution have energies above the escape energy, and this enables them to leave the planet. Since the escaping particles are neutral, Jeans escape does not depend on the magnetic field. The process is significant only for hydrogen, due to its low mass, with escape rates on the order of $10^{25} \mathrm{~s}^{-1}$ for Venus and $10^{26} \mathrm{~s}^{-1}$ for Earth and Mars. The Jeans escape rate for species $\alpha$ is (Öpik 1963)

$$
\begin{aligned}
Q_{\mathrm{Je}, \alpha} & =4 \pi r_{\text {exo }}^{2} \sqrt{\frac{k_{\mathrm{B}} T_{\text {exo }}}{2 \pi m_{\alpha}}} n_{\text {exo }, \alpha}\left(1+\frac{G M_{\text {planet }} m_{\alpha}}{r_{\text {exo }} k_{\mathrm{B}} T_{\text {exo }, \alpha}}\right) \\
& \exp \left(-\frac{G M_{\text {planet }} m_{\alpha}}{r_{\text {exo }} k_{\mathrm{B}} T_{\text {exo }}}\right),
\end{aligned}
$$

where $r_{\mathrm{exo}}, T_{\mathrm{exo}, \alpha}$, and $n_{\mathrm{exo}, \alpha}$ are the radius, temperature, and density of the exobase, respectively; $k_{\mathrm{B}}$ is Boltzmann's constant; $G$ is Newton's gravitational constant; $M_{\text {planet }}$ is the mass of the planet and $m_{\alpha}$ is the atomic mass of species $\alpha$, which represents either hydrogen or oxygen. Values of these parameters are shown in Table A.1.

\section{A.2.2. Dissociative recombination}

Dissociative recombination of molecular oxygen ions produces energetic oxygen atoms that can escape Mars but not the heavier planets Earth and Venus. The escaping atoms acquire $7 \mathrm{eV}$ or less through dissociative recombination (Cravens et al. 2017) 
Table A.1. Planetary parameters.

\begin{tabular}{|c|c|c|c|c|c|c|}
\hline Parameter & Venus & Earth & Mars & Unit & Explanation & References \\
\hline$r_{\text {planet }}$ & 6051.8 & 6371 & 3389.5 & $\mathrm{~km}$ & Mean radius of planet & \\
\hline$M_{\text {planet }}$ & $4.867 \times 10^{24}$ & $5.972 \times 10^{24}$ & $6.417 \times 10^{23}$ & $\mathrm{~kg}$ & Mass of planet & \\
\hline$n_{\mathrm{sw}}$ & $1.2 \times 10^{7}$ & $6.0 \times 10^{6}$ & $2.6 \times 10^{6}$ & $\mathrm{~m}^{-3}$ & Solar wind density & \\
\hline$v_{\mathrm{sw}}$ & $4 \times 10^{5}$ & $4 \times 10^{5}$ & $4 \times 10^{5}$ & $\mathrm{~ms}^{-1}$ & Solar wind speed & \\
\hline$B_{z}$ & -12 & -10 & -7 & $\mathrm{nT}$ & IMF $B_{z}$ & \\
\hline$r_{\text {exo }}$ & 6271.8 & 6871 & 3609.5 & $\mathrm{~km}$ & Radius of the exobase & 1,2 \\
\hline$r_{\mathrm{IMB}}$ & 6666.8 & 7647 & 4489.5 & $\mathrm{~km}$ & Radius of the IMB & 3,4 \\
\hline$m_{\mathrm{d} 0}$ & $<7 \times 10^{18}$ & $7.77 \times 10^{22}$ & $<2 \times 10^{18}$ & $\mathrm{Am}^{2}$ & Dipole moment & $5-7$ \\
\hline$n_{\mathrm{exo}, \mathrm{H}}$ & $1.3 \times 10^{9}$ & $8.5 \times 10^{10}$ & $2.5 \times 10^{10}$ & $\mathrm{~m}^{-3}$ & Hydrogen density at exobase & 8 \\
\hline$n_{\text {exo,O }}$ & $7.5 \times 10^{10}$ & $4 \times 10^{10}$ & $5.7 \times 10^{12}$ & $\mathrm{~m}^{-3}$ & Oxygen density at exobase & $9-11$ \\
\hline$T_{\text {exo,H }}$ & 1020 & 900 & 350 & $\mathrm{~K}$ & Exobase temperature & 1,8 \\
\hline$T_{\text {exo,O }}$ & 6400 & 4100 & 300 & $\mathrm{~K}$ & Exobase temperature & $10-12$ \\
\hline$Q_{0, \mathrm{pc}, \mathrm{H}}$ & - & $7.8 \times 10^{25}$ & - & $\mathrm{s}^{-1}$ & Ref. rate polar cap H escape & 13 \\
\hline$Q_{0, \mathrm{pc}, \mathrm{O}}$ & - & $8 \times 10^{24}$ & - & $\mathrm{s}^{-1}$ & Ref. rate polar cap O escape & 13,14 \\
\hline$Q_{0, \mathrm{cu}, \mathrm{H}}$ & - & $5 \times 10^{24}$ & - & $\mathrm{s}^{-1}$ & Ref. rate cusp H escape & 15 \\
\hline$Q_{0, \mathrm{cu}, \mathrm{O}}$ & - & $2 \times 10^{25}$ & - & $\mathrm{s}^{-1}$ & Ref. rate cusp $O$ escape & $15-17$ \\
\hline$Q_{0, \mathrm{pu}, \mathrm{H}}$ & $1.3 \times 10^{25}$ & $5.3 \times 10^{26}$ & $2.3 \times 10^{25}$ & $\mathrm{~s}^{-1}$ & Ref. rate pickup H escape ${ }^{a}$ & 1 \\
\hline$Q_{0, \mathrm{pu}, \mathrm{O}}$ & $1.2 \times 10^{25}$ & $7.9 \times 10^{22}$ & $2.6 \times 10^{32}$ & $\mathrm{~s}^{-1}$ & Ref. rate pickup $\mathrm{O}$ escape ${ }^{a}$ & $1,18,19$ \\
\hline$Q_{0, \mathrm{cf}, \mathrm{H}}$ & $1.4 \times 10^{25}$ & $7.7 \times 10^{25}$ & $2.0 \times 10^{24}$ & $\mathrm{~s}^{-1}$ & Cross-field ion loss rate $\mathrm{H}$ & $20-22$ \\
\hline$Q_{0, \mathrm{cf}, \mathrm{O}}$ & $5.2 \times 10^{24}$ & $3 \times 10^{24}$ & $2.0 \times 10^{24}$ & $\mathrm{~s}^{-1}$ & Cross-field ion loss rate $\mathrm{O}$ & $21-24$ \\
\hline$Q_{0, \mathrm{ldr}, \mathrm{O}}$ & 0 & 0 & $5 \times 10^{24}$ & $\mathrm{~s}^{-1}$ & Low diss. recomb. rate & 25 \\
\hline$Q_{0, \mathrm{hdr}, \mathrm{O}}$ & 0 & 0 & $5.9 \times 10^{25}$ & $\mathrm{~s}^{-1}$ & High diss. recomb. rate & 26 \\
\hline$Q_{0, \mathrm{sp}, \mathrm{O}}$ & $6 \times 10^{24}$ & $6 \times 10^{24}$ & $4.5 \times 10^{23}$ & $\mathrm{~s}^{-1}$ & Ref. rate sputtering & $27-29$ \\
\hline
\end{tabular}

Notes. The table lists the parameters used in the escape calculation. ${ }^{(a)}$ The tabulated pickup rates $Q_{0, \mathrm{pu}, \alpha}$ correspond to the hypothetical case where the boundary is at the exobase. Actual escape rates are found by scaling according to Eq. (A.10).

References. (1) Lammer (2013); (2) Lichtenegger et al. (2006); (3) Stenberg Wieser et al. (2015); (4) Bertucci et al. (2011); (5) Acuña et al. (2001); (6) Olson \& Amit (2006); (7) Luhmann et al. (2015); (8) Anderson Jr. \& Hord (1977); (9) McElroy et al. (1982); (10) Shematovich et al. (1994); (11) Chaufray et al. (2009); (12) Nagy et al. (1981); (13) Engwall et al. (2009); (14) Maes et al. (2016); (15) Pollock et al. (1990); (16) Nilsson (2011); (17) Slapak et al. (2013); (18) Masunaga et al. (2013); (19) Ramstad et al. (2015); (20) Lundin et al. (2009); (21) Nordström et al. (2013); (22) André \& Cully (2012); (23) Nilsson et al. (2011); (24) Welling et al. (2015); (25) Lammer \& Bauer (1991); (26) Cravens et al. (2017); (27) Luhmann \& Kozyra (1991); (28) Lammer et al. (2006); (29) Chaufray et al. (2007).

and that makes it difficult to directly observe the escaping flux with particle detectors. Recent estimates give an escape rate of $5.9 \times 10^{25} \mathrm{~s}^{-1}$ for this process (Cravens et al. 2017), which would make it the dominant escape process at Mars. Earlier estimates have been one order of magnitude lower (Lammer \& Bauer 1991). There is reasonable agreement on the production rates of the fast oxygen atoms. What differs between the estimates is the cross section used for collisions that may prevent the fast atoms to escape. Experimental determination of these cross sections is lacking, and the estimates are based on theoretical models. To reflect the range over which this can cause the Mars oxygen escape rates to vary, we have computed our escape rates for two different values: one low (Lammer \& Bauer 1991) $Q_{0,1 \mathrm{ld}, \mathrm{O}}$ (dotted lines in Figs. A.1 and 2) and one high (Cravens et al. 2017) $Q_{0, \text { hdro }}$ (solid lines). As these values do not depend on the planetary magnetic field they do not affect the conclusions on the magnetic field dependence of the total escape rates.

\section{A.2.3. Cross-field ion loss}

Cross-field ion loss includes the plasmaspheric wind and plumes on magnetised planets (Lemaire \& Schunk 1992; André \& Cully 2012) and ions being lost directly from the ionosphere at the unmagnetised planets (Lundin et al. 2008; Nordström et al. 2013; Edberg et al. 2011). On unmagnetised planets, the ions drift across magnetic field lines on the dayside and the nightside contribution is insignificant (Fränz et al. 2015). Although nightside escape may be better described as unmagnetised rather than cross-field, we include it here as it is too insignificant to warrant a category of its own. Loss from the polar caps is not included in cross-field ion loss, since that plasma escapes without moving across magnetic field lines and is accounted for under polar cap escape. At magnetised planets a dense plasmasphere is built up, which is subsequently lost through the dayside magnetopause or the tail via plasmaspheric plumes or a plasmaspheric wind. While this leads to an escape that is highly variable with time, the average escape rates depend on the ionospheric conditions. For current conditions, the solar activity ensures sufficient crossfield transport so that the average escape rates can be modelled as constants multiplied by the fraction of the ionospheric surface area that is outside the polar cap:

$Q_{\mathrm{cf}, \alpha}=Q_{0, \mathrm{cf}, \alpha} \frac{1-\frac{\Omega_{\mathrm{pc}}}{4 \pi}}{1-\frac{\Omega_{\mathrm{pc}, \mathrm{planet}}}{4 \pi}}$,

where $\Omega_{\mathrm{pc}}$ is the solid angle of the polar caps combined for the hypothetical Venus-like, Earth-like, and Mars-like planets, and $\Omega_{\mathrm{pc} \text {,planet }}$ is the solid angle of the polar caps combined for these planets today, that is, zero for Mars and Venus and $0.63 \mathrm{sr}$ for Earth. The specific constants, $Q_{0, \mathrm{cf}, \mathrm{H}}$ and $Q_{0, \mathrm{cf}, \mathrm{O}}$ are tabulated in Table A.1. The magnetic field dependence introduced in 


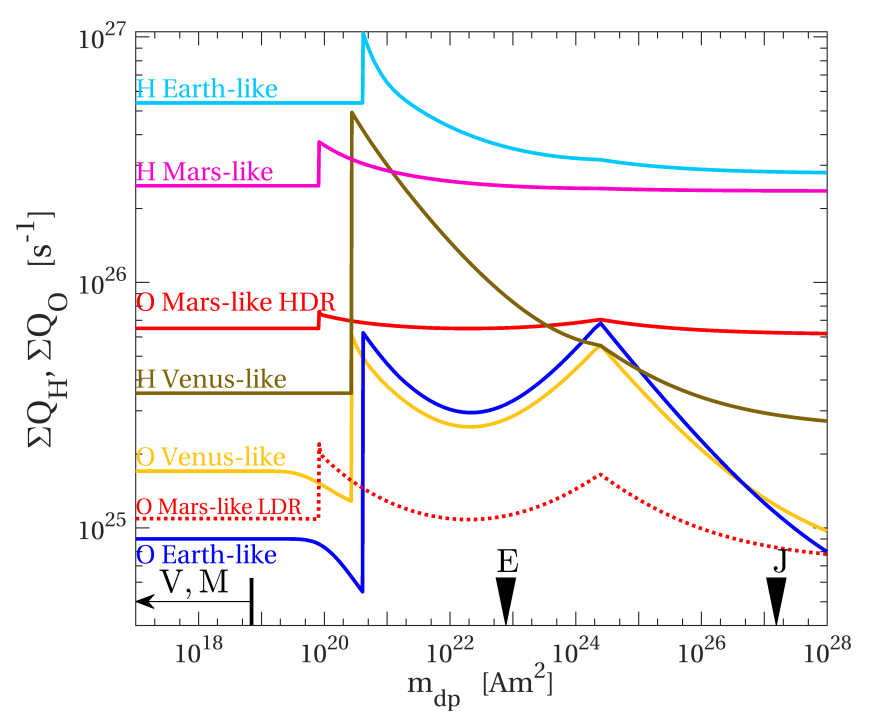

Fig. A.1. Hydrogen and oxygen escape rates for Venus-like, Earth-like, and Mars-like planets. For oxygen escape from Mars, the two curves represent high (HDR) and low (LDR) dissociative recombination estimates. The magnetic moments of present-day Earth and Jupiter are marked on the horizontal axis for reference. The horizontal arrow indicates the unmagnetised character of Venus and Mars.

Eq. (A.7) does not significantly affect the total escape rates that include all processes.

\section{A.2.4. Ion pickup}

The pickup ion escape rate is proportional to the number of neutrals in the exosphere that are present outside the magnetopause or IMB. We assume a spherically symmetric exosphere in which the density is proportional to $\exp \left(-r / h_{\alpha}\right)$, where

$h_{\alpha}=\frac{k_{\mathrm{B}} T_{\mathrm{exo}} r_{\mathrm{exo}}^{2}}{G M_{\text {planet }} m_{\alpha}}$

is the scale height for species $\alpha$. The number of neutral particles of species $\alpha$ outside a radius $r$ is proportional to

$N_{\alpha}(\infty)-N_{\alpha}(r)$,

where the function $N_{\alpha}(r)$ is defined

$$
\begin{aligned}
& N_{\alpha}(r)=\int_{0}^{r} 4 \pi r^{2} \mathrm{e}^{-\frac{r^{\prime}}{h_{\alpha}}} \mathrm{d} r^{\prime}= \\
& 8 \pi h_{\alpha}^{3}-4 \pi\left(2 h_{\alpha}^{3}+2 h_{\alpha}^{2} r+h_{\alpha} r^{2}\right) \mathrm{e}^{-\frac{r}{h_{\alpha}}} .
\end{aligned}
$$

Allowing $Q_{0, \mathrm{pu}, \alpha}$ to be the pickup escape rate when the boundary, that is, MP or IMB, is at the exobase, and $r_{\mathrm{b}}$ the planetocentric distance of that boundary, the escape rate for ion pickup is

$$
\begin{gathered}
Q_{\mathrm{pu}, \alpha}=Q_{0, \mathrm{pu}, \alpha} \frac{N_{\alpha}(\infty)-N_{\alpha}\left(r_{\mathrm{b}}\right)}{N_{\alpha}(\infty)-N_{\alpha}\left(r_{\mathrm{exo}}\right)}= \\
Q_{0, \mathrm{pu}, \alpha} \frac{2 h_{\alpha}^{3}+2 h_{\alpha}^{2} r_{\mathrm{b}}+h_{\alpha} r_{\mathrm{b}}^{2}}{2 h_{\alpha}^{3}+2 h_{\alpha}^{2} r_{\mathrm{exo}}+h_{\alpha} r_{\mathrm{exo}}^{2}} \mathrm{e}^{\frac{r_{\mathrm{exo}-r_{\mathrm{b}}}}{h_{\alpha}}} .
\end{gathered}
$$

The meaning of the constant $Q_{0, \mathrm{pu}, \alpha}$ in Eq. (A.10) is the escape rate for the hypothetical case where the boundary is at the exobase. The values of $Q_{0, p u, \alpha}$ in Table A.1 are scaled so that Eq. (A.10) yields the observed escape rates when $r_{\mathrm{b}}$ is equal to the observed standoff distances of the boundaries at Mars and Venus. For Earth, the magnetic field prevents measurements that could be used for Earth-like planets. Therefore, we instead scale the Venus value,

$$
Q_{0, \mathrm{pu}, \alpha, \mathrm{E}}=Q_{0, \mathrm{pu}, \alpha, \mathrm{V}} \cdot \frac{n_{\mathrm{exo}, \alpha, \mathrm{E}} \cdot \mathrm{e}^{-\frac{r_{\mathrm{IMB}, \mathrm{E}}-r_{\mathrm{exo}, \mathrm{E}}}{h_{\alpha, \mathrm{E}}}}}{n_{\mathrm{exo}, \alpha, \mathrm{V}} \cdot \mathrm{e}^{-\frac{r_{\mathrm{IMB}, \mathrm{V}}-r_{\mathrm{exo}, \mathrm{V}}}{h_{\alpha, \mathrm{V}}}}},
$$

where $\mathrm{E}$ and $\mathrm{V}$ in the subscripts mean Earth and Venus, respectively.

\section{A.2.5. Sputtering}

Sputtering is proportional to the ratio of the number of neutrals within one gyroradius $r_{\mathrm{g}}$ above the exobase to the total number of neutrals above the exobase. We have

$$
\begin{aligned}
& Q_{\mathrm{sp}, \mathrm{O}}=Q_{0, \mathrm{sp}, \mathrm{O}} \frac{\int_{r_{\text {exo }}}^{r_{\text {exo }}+r_{\mathrm{g}}} r^{2} \mathrm{e}^{-\frac{r}{h_{\mathrm{O}}}} \mathrm{d} r}{\int_{r_{\mathrm{exo}}}^{\infty} r^{2} \mathrm{e}^{-\frac{r}{h_{\mathrm{O}}}} \mathrm{d} r} \\
& =Q_{0, \mathrm{sp}, \mathrm{O}}\left(1-\frac{2 h_{\mathrm{O}}^{2}+2 h_{\mathrm{O}}\left(r_{\mathrm{exo}}+r_{\mathrm{g}}\right)+\left(r_{\mathrm{exo}}+r_{\mathrm{g}}\right)^{2}}{2 h_{\mathrm{O}}^{2}+2 h_{\mathrm{O}} r_{\text {exo }}+r_{\mathrm{exo}}^{2}} \mathrm{e}^{-\frac{r_{\mathrm{g}}}{h_{\mathrm{O}}}}\right) .
\end{aligned}
$$

The energy of the impacting ions depends on the solar wind properties and the size of the magnetosphere. We have assumed that $1 \mathrm{keV} \mathrm{O}^{+}$ions are typical. Luhmann \& Kozyra (1991) showed that the distribution functions of the impacting ions decrease rapidly above that energy. The reason is that for ions to reach high energies they must be accelerated over long distances and therefore start their journey farther away from the planet where the source density is small. As the magnetosphere grows the convective electric field of the solar wind is shielded from the inner magnetosphere, further contributing to the falloff of the sputtering rate with magnetic moment. We have not attempted to model this effect, as it would modify the result only in a region where sputtering escape is already insignificant. The sputtering reference rate for Mars in Table A. 1 is the mean of the solar minimum and maximum rates (Chaufray et al. 2007). There are no estimates for Earth in the literature since sputtering is prevented by the magnetic field. We have used the Venus sputtering escape rate (Luhmann \& Kozyra 1991; Lammer et al. 2006) also for Earth, because Earth and Venus are similar in size, mass, and exosphere temperature.

Sputtering in the auroral zone on Earth has been estimated at $8 \times 10^{23} \mathrm{~s}^{-1}$ (Shematovich et al. 2006). We use this value scaled by the square of the exobase radius,

$8 \times 10^{23} \mathrm{~s}^{-1}\left(\frac{r_{\text {exo }}}{r_{\text {exo,Earth }}}\right)^{2}$,

as a lower limit to sputtering escape (Fig. 3). This estimate does not take any of the auroral physics into account, but at $8 \times 10^{23} \mathrm{~s}^{-1}$, or below, the contribution to the total escape rates is negligible.

\section{A.2.6. Polar cap escape}

The rate of escape from the polar cap is proportional to the polar cap area, and therefore to $\Omega_{\mathrm{pc}} r_{\mathrm{exo}}^{2}$, the solid angle of the polar cap multiplied by the exobase radius squared. The escape rate for present-day Earth, $Q_{0, \mathrm{pc}, \alpha}$, is found in Table A.1. For other 
planets and magnetic moments, the rate is scaled in proportion to $\Omega_{\mathrm{pc}} r_{\mathrm{exo}}^{2}$.

$Q_{\mathrm{pc}, \alpha}=Q_{0, \mathrm{pc}, \alpha} \frac{\Omega_{\mathrm{pc}}}{\Omega_{\mathrm{pc}, \mathrm{E}}}\left(\frac{r_{\mathrm{exo}}}{r_{\mathrm{exo}, \mathrm{E}}}\right)^{2}$.

Escape rates for the individual processes that depend on magnetic moment are shown in Fig. 3.

\section{A.2.7. Cusp escape}

Kinetic energy of the plasma that enters the cusp from the dayside magnetopause is transferred to wave energy through plasma instabilities. Energy is also transferred from the solar wind to the low altitude cusp by Poynting flows (Strangeway et al. 2005). This energy heats the plasma at low altitude, causing it to escape as perpendicular kinetic energy is converted to parallel energy by the magnetic mirror force. The cusp escape rate behaves differently for low and high magnetic moments. At low magnetic moments, the escape rate from the cusp is proportional to the available solar wind energy, which is proportional to the cross section of the magnetosphere, $\pi r_{\mathrm{c}}^{2}$. The upgoing ion flux is limited by what the ionosphere can supply (Barakat et al. 1987), causing saturation for high magnetic moments. For all magnetic moments, the escape rate is proportional to the area of the cusp, which is proportional to the solid angle of the polar cap and the radius of the exobase squared. Therefore, we have, in total,

$Q_{\mathrm{cu}, \alpha}=\min \left(Q_{0, \mathrm{cu}, \alpha}\left(\frac{r_{\mathrm{c}}}{r_{\mathrm{c}, \mathrm{E}}}\right)^{2}, Q_{\mathrm{max}, 0, \mathrm{cu}, \alpha}\right) \cdot \frac{\Omega_{\mathrm{pc}}}{\Omega_{\mathrm{pc}, \mathrm{E}}}\left(\frac{r_{\mathrm{exo}}}{r_{\mathrm{exo}, \mathrm{E}}}\right)^{2}$,

where $Q_{0, \mathrm{cu}, \alpha}$ is the cusp escape rate for present-day Earth, tabulated in Table A.1. For Earth, the limiting rates were found by Barakat et al. (1987) by computing ionisation and vertical transport in the ionosphere, and these limiting rates are $Q_{\max , 0, \mathrm{cu}, \mathrm{H}}=$ $5 \times 10^{25} \mathrm{~s}^{-1}$ for hydrogen and $Q_{\max , 0, \mathrm{cu}, \mathrm{O}}=2 \times 10^{26} \mathrm{~s}^{-1}$ for oxygen, that is, ten times higher than the average rates in Table A.1. For Venus and Mars, we assume that the flux is the same as for Earth, and the limiting escape rates scale with the factor

$$
\frac{\Omega_{\mathrm{pc}}}{\Omega_{\mathrm{pc}, \mathrm{E}}}\left(\frac{r_{\mathrm{exo}}}{r_{\mathrm{exo}, \mathrm{E}}}\right)^{2}
$$

in Eq. (A.14). Scaling in this way introduces an uncertainty. However, the ionospheric electron densities and the composition of the escaping plasma are similar at the three planets - the $\mathrm{H}^{+}$to $\mathrm{O}^{+}$ratio of the escaping plasma is about 1 for Mars (Lundin et al. 2009), 2 for Venus (Barabash et al. 2007), and 0.25 for Earth (Table A.1) - and therefore the scaling procedure provides a reasonable approximation of the value and position of the peak at cusp outflow saturation. Modelling the escape rate according to Eq. (A.14), we assume that all ions leaving the cusp escape and that the return flows are negligible, which is consistent with observations by the Cluster spacecraft (Nilsson 2011; Nilsson et al. 2012; Slapak et al. 2017a,b).

\section{A.2.8. Other means of escape}

There are mechanisms that we have not included in our model. Ions that are present on magnetospheric field lines that are opened by reconnection at the dayside magnetopause may escape directly. Some of these are observed by the spacecraft that measure outflow from the cusp and they are therefore automatically included in the estimates. Ions located closer to the reconnection site may escape directly, but their contribution is not expected to be significant due to the low density in the region.

Unlike particles originating in the cusps and polar caps, which can escape directly, outflows from the auroral zone occur on closed field lines. Outflowing ions may meet different fates: they may return to the ionosphere, as in regions with outflow there is also precipitation (Newell et al. 2009), or they may drift across field lines and escape through the magnetopause or along the magnetotail. While further research is needed to determine the rates of escape and precipitation from the plasma sheet, these processes can only add to the escape rates at magnetised planets, and contributions from them do not change the conclusions of this paper.

We treat Mars as a completely unmagnetised planet. This is a simplification, since there are crustal fields that cause magnetic loops to be present in the Martian southern hemisphere inside the IMB. Although there is a possibility that such magnetic loops may affect escape rates, Mars Express observations (Nilsson et al. 2006) showed only an insignificant influence on the detected planetary ions. 